\title{
Sensitivity of intrinsic low-dimensional manifolds with respect to kinetic data
}

\author{
Karin König*, Ulrich Maas \\ Institut für Technische Thermodynamik, Karlsruhe University (TH), Kaiserstrasse 12, D-76128 Karlsruhe, Germany
}

\begin{abstract}
In this paper, a mathematical model is introduced for the calculation of sensitivities of Intrinsic LowDimensional Manifolds (ILDMs) with respect to kinetic data. This model allows treatment of questions that have not yet been discussed in sufficient detail in the context of ILDM: Which reactions are governing the ILDM, are they the same reactions that govern the detailed mechanism, and how does the ILDM change due to changes in the kinetic data in the underlying detailed mechanism? Based on the governing equation for the ILDMs, sensitivity equations are derived by partial differentiation with respect to kinetic parameters. Special numeric techniques are applied to allow a scaling-invariant calculation of the underlying Jacobian matrices. Some sample calculations are introduced for the stoichiometric $\mathrm{CO} / \mathrm{H}_{2} / \mathrm{O}_{2} / \mathrm{N}_{2}$ system to validate the approach. The examples shown in the paper also give some first impressions of the values of the sensitivities for some important reactions and they show the sensitive reactions within the sample system. In this context of results, the sensitivity of an ILDM is compared to the sensitivity of a Perfectly Stirred Reactor (PSR).
\end{abstract}

(C) 2004 The Combustion Institute. Published by Elsevier Inc. All rights reserved.

Keywords: Reduced mechanisms; Chemical kinetics; Sensitivity analysis

\section{Introduction}

In the past years, interest in the simulation of combustion systems has considerably increased, mainly in view of an efficient, clean operation of practical combustion devices. Numerical simulations of combustion processes based on detailed kinetic models have become a standard research tool for 1- and 2-dimensional systems, and the chemical kinetics needed for these simulations is well known [1] and can be used in its detailed form. But in many cases it is still computationally prohibitive [1] to use detailed chemistry for the

\footnotetext{
* Corresponding author. Fax: +497216083931.

E-mail address: koenig@itt.mach.uni-karlsruhe.de (K. König).
}

simulation of turbulent reactive flows in 3 dimensions. This originates from the huge reaction mechanisms, which often consist of more than 1000 species and thousands of elementary reactions (for example, the low temperature oxidation of higher hydrocarbons [2]). To allow simulations of 3-dimensional systems with a reasonable computational effort, it is necessary to reduce the chemical kinetics. One of the large number of methods for mechanism reduction is the method of Intrinsic Low-Dimensional Manifolds (ILDM) $[3,4]$ that allows a description of the complex kinetics. The method is based on an analysis of the governing time scales in the reacting system. In the past, a lot of work has been done to improve and to extend the methodology (see, e.g. [5-8] and references therein). Recently, some

1540-7489/\$ - see front matter (c) 2004 The Combustion Institute. Published by Elsevier Inc. All rights reserved. doi:10.1016/j.proci.2004.08.217 
works addressed the influence of the detailed kinetics. The effect of adding fast reactions to chemical systems was investigated in [9], and the influence of the addition of slow reactions was analysed in [10]. Of course, the quality of the reduced kinetics depends on the quality of the detailed mechanism it is based on. On the other hand, the rate coefficients in the detailed mechanisms are subject to uncertainties. To determine which reactions are very important in the detailed mechanism, sensitivity analyses of the detailed mechanisms have been developed and extensively used. Consequently, the question appears which reactions are important for the ILDMs. In other words, it is necessary to know how the ILDM, i.e., the simplified kinetics, changes, when the detailed reaction mechanism is modified, e.g., when the rate coefficients are subject to changes, and it is also fundamental to know, which reactions are governing the ILDM. Are they the same reactions that are of importance in detailed kinetics? Does sensitivity analysis of ILDMs allow one to approximate an ILDM that corresponds to a detailed mechanism where the rate coefficients have changed?

These questions have not yet been addressed in sufficient detail in the context of ILDM. Admittedly, sensitivities of slow manifolds with respect to perturbations have been discussed before, for example in [8], where a formulation for sensitivity analysis of Attracting Low-Dimensional Manifolds (ALDMs) is discussed. These ALDMs are constructed on the base of a geometrical simplification of the chemical kinetics and represent global attractors, unlike ILDMs, which are local attractors.

To find an answer to the above-named questions for ILDMs, a mathematical model for the calculation of sensitivities of ILDMs has been developed and shall be presented in this paper. Some results for a stoichiometric $\mathrm{CO} / \mathrm{H}_{2} / \mathrm{O}_{2} / \mathrm{N}_{2}$ system are discussed to check the validity of the model and to show an example of sensitivity values and their dependence on the domain of the ILDM. Furthermore, the relation between the sensitivity analysis of a Perfectly Stirred Reactor (PSR) and the sensitivity analysis of an ILDM is discussed.

\section{Mathematical model}

To derive the sensitivity equations, we start from the definition of the ILDMs and adopt a notation similar to [11]. Let $\psi$ denote the $n$-dimensional vector of state variables, and let the dynamical system be governed by the ordinary differential equation system

$$
\frac{\partial \boldsymbol{\psi}}{\partial t}=\boldsymbol{F}(\boldsymbol{\psi} ; \boldsymbol{p})
$$

where $t$ denotes the time and $\boldsymbol{F}(\boldsymbol{\psi})$ the vector valued function of the chemical rates of change. $p$ denotes the $n_{\mathrm{p}}$-dimensional vector of parameters governing the system, such as the rate coefficients. Following the notation in [11], we use the partial derivative of $\psi$ with respect to the time, because this allows an easy addition of terms for physical processes, even though the only processes considered here are chemical ones, and therefore $\psi$ is a function of time only. To simplify the notation, we consider as follows the dependence on one parameter $p$ only without a loss of generality. For an arbitrary number of different parameters, the resulting system of equations has to be solved simply for one perturbed parameter after the other. Following Maas [11], we decompose the Jacobian matrix $\boldsymbol{F}_{\boldsymbol{\psi}}$ of the system into slow and fast subspaces, respectively:

$$
\left(\begin{array}{c}
\tilde{Z}_{\mathrm{s}} \\
\tilde{Z}_{\mathrm{f}}
\end{array}\right) \cdot \boldsymbol{F}_{\psi}=\left(\begin{array}{cc}
N_{\mathrm{s}} & 0 \\
\mathbf{0} & N_{\mathrm{f}}
\end{array}\right) \cdot\left(\begin{array}{c}
\tilde{Z}_{\mathrm{s}} \\
\tilde{Z}_{\mathrm{f}}
\end{array}\right),
$$

where $N_{\mathrm{s}}$ is an $\left(n_{\mathrm{s}} \times n_{\mathrm{s}}\right)$-dimensional matrix (with $n_{\mathrm{s}}$ denoting the number of slow processes) and $\boldsymbol{N}_{\mathrm{f}}$ is an $\left(n_{\mathrm{f}} \times n_{\mathrm{f}}\right)$-dimensional matrix (with $n_{\mathrm{f}}$ denoting the number of fast processes). The eigenvalues are ordered according to $\lambda^{\text {real }}\left(\boldsymbol{N}_{\mathrm{s}}\right)>\lambda^{\text {real }}\left(\boldsymbol{N}_{\mathrm{f}}\right) . \tilde{\boldsymbol{Z}}_{\mathrm{f}}$ and $\tilde{\boldsymbol{Z}}_{\mathrm{s}}$ are left invariant subspaces that span the same space as the fast and slow eigenvectors, respectively. Using this notation, the ILDM of the system is given by

$\tilde{\boldsymbol{Z}}_{\mathbf{f}}(\boldsymbol{\psi} ; p) \cdot \boldsymbol{F}(\boldsymbol{\psi} ; p)=\boldsymbol{G}(\boldsymbol{\psi} ; p)=0$,

which is an $n_{\mathrm{f}}$-dimensional equation system for $n$ unknowns. Note that $\boldsymbol{F}$ is not only a function of $\psi$, but also of the parameter $p$ (e.g., a rate coefficient of a reaction) and thus, $\tilde{Z}_{\mathbf{f}}$ depends on $\psi$ as well as on $p$, too.

Our aim is now to know how a change in the parameter $p$ influences the ILDM. Differentiation of $\boldsymbol{G}$ yields

$\boldsymbol{G}_{\psi} \cdot \frac{\partial \psi}{\partial p}+\boldsymbol{G}_{p}=0$,

where $\boldsymbol{G}_{\psi}$ denotes the $\left(n_{\mathrm{f}} \times n\right)$-dimensional matrix of partial derivatives of $\boldsymbol{G}$ with respect to $\psi$, and $\boldsymbol{G}_{p}$ denotes the $n_{\mathrm{f}}$-dimensional vector of the partial derivatives of $\boldsymbol{G}$ with respect to $p$. This is an underdetermined $n_{\mathrm{f}}$-dimensional equation system for the $n$-dimensional vector $\partial \psi / \partial p$ of sensitivities. The reason for the equation system being underdetermined is that only components of the sensitivities not tangential to the ILDM are obtained from this equation. It is important to note that all components of the sensitivities tangential to the ILDM would not yield a change of the ILDM itself. This means that the sensitivity can be decomposed, resulting in a component representing the perpendicular displacement of the ILDM and another component describing the perturba- 
tion of the movement along the ILDM. This issue of the two components of such sensitivities is thoroughly discussed in [8]. Because all components of the sensitivities tangential to the manifold do not change the ILDM itself, we express the vector of sensitivities as a linear combination of $n_{\mathrm{f}}$ vectors perpendicular to the ILDM. These vectors are for the implicitly defined manifold (Eq. (3)), given as the transposed of the matrix of partial derivatives of $G$ with respect to $\psi$. Therefore, the vector $\partial \hat{\psi} / \partial p$ of sensitivities perpendicular to the manifold (which we shall simply call "sensitivity of the ILDM" as follows) can be expressed by:

$\boldsymbol{S}=\frac{\partial \hat{\boldsymbol{\psi}}}{\partial p}=\boldsymbol{G}_{\psi}^{\mathrm{T}} \cdot \boldsymbol{x}$

with $\hat{\psi}$ representing the projected vector of state variables perpendicular to the ILDM. Combining Eqs. (4) and (5) yields an equation system for $\boldsymbol{x}$ :

$\boldsymbol{G}_{\boldsymbol{\psi}} \cdot \boldsymbol{G}_{\psi}^{\mathrm{T}} \cdot \boldsymbol{x}+\boldsymbol{G}_{p}=0$,

$\boldsymbol{x}=-\left(\boldsymbol{G}_{\psi} \cdot \boldsymbol{G}_{\psi}^{\mathrm{T}}\right)^{-1} \cdot \boldsymbol{G}_{p}$

and inserting Eq. (7) in Eq. (5), we obtain for $\boldsymbol{S}$

$\boldsymbol{S}=-\boldsymbol{G}_{\psi}^{\mathrm{T}} \cdot\left(\boldsymbol{G}_{\psi} \cdot \boldsymbol{G}_{\psi}^{\mathrm{T}}\right)^{-1} \cdot \boldsymbol{G}_{p}=-\boldsymbol{G}_{\psi}^{+} \cdot \boldsymbol{G}_{p}$,

where $\boldsymbol{G}_{\psi}^{+}$denotes the Moore-Penrose pseudo inverse of $\boldsymbol{G}_{\boldsymbol{\psi}}$. The invertibility of the product $\boldsymbol{G}_{\boldsymbol{\psi}}^{\mathrm{T}} \cdot \boldsymbol{G}_{\boldsymbol{\psi}}$ is warranted, because the matrix $\boldsymbol{G}_{\boldsymbol{\psi}}$ is of full rank $n_{\mathrm{f}}$ for an $\left(n \times n_{\mathrm{f}}\right)$-dimensional manifold, and therefore the Moore-Penrose pseudo inverse can be calculated according to Eq. (8) [12]. Eq. (8) allows in principle to calculate the sensitivity of the ILDM at any arbitrary point of the ILDM. Numerical problems arise, however, due to the fact that the numerical algorithms for the determination of invariant subspaces yield the subspace $\tilde{Z}_{\mathrm{f}}$ with an arbitrary scaling, which makes it impossible to approximate $\boldsymbol{G}_{\boldsymbol{\psi}}$ and $\boldsymbol{G}_{p}$ by finite differences. Eq. (3) for the ILDM can be rewritten by replacing $\tilde{Z}_{\mathrm{f}}$ by a scaling invariant formulation to overcome the problem

$$
\hat{\boldsymbol{G}}=\left(\tilde{\boldsymbol{Z}}_{\mathbf{f}}(\boldsymbol{\psi} ; p) \cdot \boldsymbol{P}\right)^{-1} \cdot \tilde{\boldsymbol{Z}}_{\mathbf{f}}(\boldsymbol{\psi} ; p) \cdot \boldsymbol{F}(\boldsymbol{\psi} ; p) .
$$

In this equation, the matrix $\boldsymbol{P}$ can be chosen arbitrarily, provided that the resulting matrix $\tilde{Z}_{\mathbf{f}}(\boldsymbol{\psi}) \cdot \boldsymbol{P}$ is regular. It can be shown by simple calculus that in this case $\hat{\boldsymbol{G}}$ is invariant with respect to an arbitrary scaling of the matrix $\tilde{Z}_{\mathrm{f}}$.

The matrices $\boldsymbol{G}_{\boldsymbol{\psi}}$ and $\boldsymbol{G}_{p}$ of partial derivatives of $\boldsymbol{G}$ are in this work calculated based on a finite difference approximation. The procedure yields a rather high number of required operations and therefore some data about the computational costs should be mentioned here. Computation of the 2-dimensional ILDM of the syngas-air-system (sample system considered in Section 3) without the use of a 1-dimensional ILDM as a starting solution takes about $99 \mathrm{~s}$ on a PC with an Intel processor, whereas the calculation of the appropriate sensitivities on each point of the ILDM needs $35 \mathrm{~s}$, what is roughly $40 \%$ of the calculation time for the ILDM itself. It should be pointed out that sensitivities can also be obtained by a perturbation analysis combined with an analytical differentiation [13], which is expected to clearly reduce the computational effort.

Summarizing, it can be stated that the algorithm presented above allows one to calculate the sensitivity of any point on the ILDM with respect to an arbitrary parameter (e.g., a rate coefficient). In other words: Eq. (8) gives the vector $\boldsymbol{S}$, which describes the displacement $\partial \hat{\boldsymbol{\psi}} / \partial p$ perpendicular to the ILDM if the parameter $p$ is perturbed, for any point $\psi$ on the ILDM. This has been performed for the ILDMs of the $\mathrm{CO} / \mathrm{H}_{2} /$ $\mathrm{O}_{2} / \mathrm{N}_{2}$-system. The results are presented in the following section.

\section{Results and discussion}

Based on the mathematical model presented above, we have investigated the sensitivity of an ILDM with respect to the kinetic parameters of the detailed reaction mechanism. The system we consider therefore is the $\mathrm{CO} / \mathrm{H}_{2} / \mathrm{O}_{2} / \mathrm{N}_{2}$-system, which consists of 13 species that react in 67 elementary reactions. The temperature of the unburnt gases is $298 \mathrm{~K}$, and the mixture is stoichiometric with $40 \mathrm{vol} \% \mathrm{CO}, 30 \mathrm{vol} \% \mathrm{H}_{2}$, and $30 \mathrm{vol} \%$ of $\mathrm{N}_{2}$ in the syngas. This system was chosen because it has been analyzed well in the context of the ILDM-concept in a previous publication [11]. Because the main aim of this paper is the derivation of the mathematical model, this system has some other positive aspects, namely that it allows (due to its simplicity) to focus the attention on the principles of the calculation method, whereas it should be pointed out that the algorithm provided can be used for arbitrarily complex systems.

To clarify the physical meaning of the calculated sensitivities, results of a sensitivity analysis for a 1d-system with respect to the rate of the reaction $\mathrm{H}+\mathrm{O}_{2}+\mathrm{M} \rightarrow \mathrm{HO}_{2}+\mathrm{M}$ are shown in Fig. 1, where the 1d-ILDM of the system is plotted together with the sensitivity vectors in the ILDM points. Note that in the following always relative sensitivities with respect to the kinetic parameters are given with $\boldsymbol{S}=\partial \hat{\boldsymbol{\psi}} / \partial \ln k$. The magnitude of the arrows in Fig. 1 is calculated such that the length directly reflects a change in $\hat{\boldsymbol{\psi}}$ by $\Delta \hat{\psi}=\boldsymbol{S} \cdot \Delta p$, where $\Delta p=\Delta \ln k=0.5$. Furthermore, an ILDM is shown, which has been calculated based on the detailed mechanism with a rate coefficient of the reaction $\mathrm{H}+\mathrm{O}_{2}+\mathrm{M} \rightarrow$ $\mathrm{HO}_{2}+\mathrm{M}$ set to $150 \%$ of its original value, corresponding to a perturbation of $+50 \%$. As it can be 


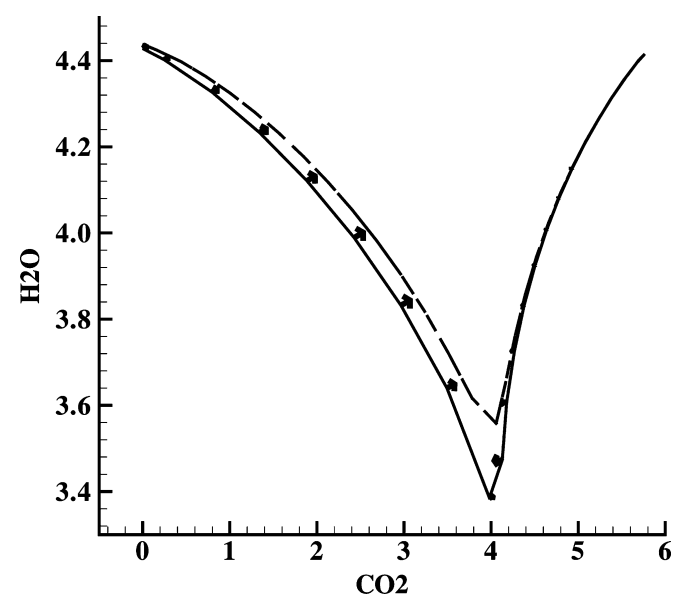

Fig. 1. 1d-ILDM of the stoichiometric $\mathrm{CO} / \mathrm{H}_{2} / \mathrm{O}_{2} / \mathrm{N}_{2-}$ system shown as a projection of the state space into the $\mathrm{CO}_{2}-\mathrm{H}_{2} \mathrm{O}$-plane. Solid line: 1d-ILDM, vectors: calculated sensitivities for this ILDM with respect to the reaction $\mathrm{H}+\mathrm{O}_{2}+\mathrm{M} \rightarrow \mathrm{HO}_{2}+\mathrm{M}$, dashed line: 1dILDM calculated with a $50 \%$ higher rate coefficient of the reaction $\mathrm{H}+\mathrm{O}_{2}+\mathrm{M} \rightarrow \mathrm{HO}_{2}+\mathrm{M}$. Species concentrations and sensitivities are given in terms of $\Phi=w_{\mathrm{i}} / M_{\mathrm{i}}$, where $w_{\mathrm{i}}$ denotes the species mass fractions and $M_{\mathrm{i}}$ the molar masses $\left(\left[w_{\mathrm{i}} / M_{\mathrm{i}}\right]=\mathrm{mol} / \mathrm{kg}\right)$.

seen, a good agreement is obtained. The endpoints of the sensitivity vectors and the points of the ILDM calculated on base of the perturbed detailed mechanism correspond very well. Differences as they can be seen, for example, at $\Phi_{\mathrm{CO}_{2}} \approx 4.0 \mathrm{~mol} / \mathrm{kg}$ can be attributed to two facts: First, for a $50 \%$ perturbation of the detailed mechanism a linear sensitivity analysis is only a rough approximation and, second, this is a domain, where the time scale separation is small and the ILDM is very sensitive with respect to perturbations (cf. [11]). Figure 2, which shows the sensitivity vectors for a $2 \mathrm{~d}-\mathrm{ILDM}$ of the system, gives a further illustration of the meaning of the sensitivity vectors. Note that in this example the scaling of the vectors has been chosen to allow a good visualization only, but, nevertheless, all vectors use the same scaling factor and thus a direct comparison of the magnitude of the perturbation in different domains is possible. Several features can be seen from this figure. First, the sensitivities clearly depend on the local domain of the combustion process. They are large in domains far from equilibrium (small values of $\mathrm{CO}_{2}$ and $\mathrm{H}_{2} \mathrm{O}$ ). This is evident because the reaction $\mathrm{H}+\mathrm{O}_{2}+\mathrm{M} \rightarrow$ $\mathrm{HO}_{2}+\mathrm{M}$ is important only for low temperatures, whereas it loses its importance for conditions close to equilibrium with their high temperatures.

Furthermore, it can be seen that directions of the sensitivity vectors can significantly change over the domain of the ILDM. An increase in the reactive rate may increase the value of $\mathrm{OH}$

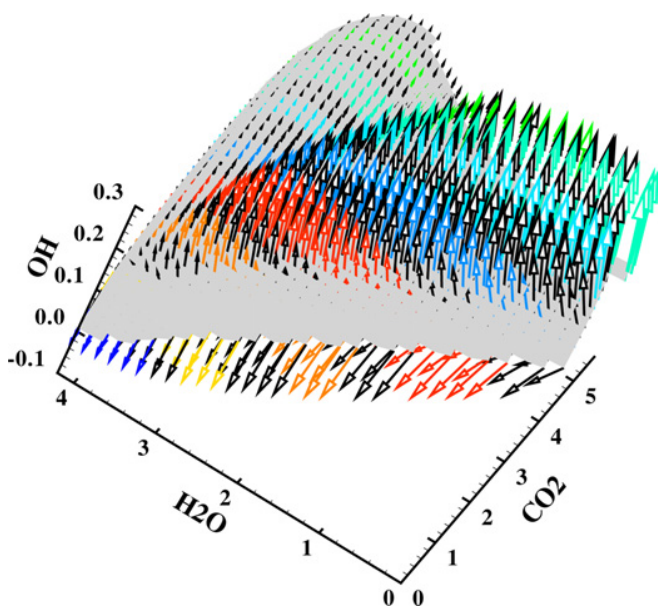

Fig. 2. 2d-ILDM - shown as grey shade - of a stoichiometric $\mathrm{CO} / \mathrm{H}_{2} / \mathrm{O}_{2} / \mathrm{N}_{2}$-system shown as projection of the state space in the $\mathrm{CO}_{2}-\mathrm{H}_{2} \mathrm{O}-\mathrm{OH}$-space. The multicolor vectors represent the calculated sensitivities with respect to the reaction $\mathrm{H}+\mathrm{O}_{2}+\mathrm{M} \rightarrow \mathrm{HO}_{2}+\mathrm{M}$. The length of the vectors was scaled with a common factor for better visualization, the colors were also chosen to allow a better visualization only. Species concentrations and sensitivities are given in terms of $\left[w_{\mathrm{i}} /\right.$ $\left.M_{\mathrm{i}}\right]=\mathrm{mol} / \mathrm{kg}$.

in some parts of the domain, whereas it may decrease it in others.

Let us now focus on a detailed discussion of the results in the context of the well-known results of sensitivity analyses with respect to the rates of elementary reactions for detailed combustion simulations (see, e.g. [14] for references). An important question concerning the ILDMs is, as mentioned in Section 1, whether the simplified kinetics depends on the same reactions as the detailed mechanism. To find an answer, let us now have a look at the common features of the sensitivity of ILDMs and the known sensitivity analysis for detailed combustion simulations. As an example, we compare the sensitivity of a PSR and the sensitivity of an ILDM. Notifications used are consistent with the ones used in the section before. A PSR in steady state is governed by

$0=\boldsymbol{F}(\boldsymbol{\psi}, p)+\omega \cdot\left(\boldsymbol{\psi}^{\text {mix }}-\boldsymbol{\psi}\right)$,

where $\omega$ denotes a characteristic mixing scale (corresponding to a residence time) and $\psi^{\text {mix }}$ the inflow composition. Therefore, the sensitivity of the PSR solution $\boldsymbol{S}^{\mathrm{PSR}}$ can be calculated as

$\boldsymbol{S}^{\mathrm{PSR}}=\frac{\partial \boldsymbol{\psi}}{\partial p}=-\left(\boldsymbol{F}_{\psi}-\omega \cdot \boldsymbol{I}\right)^{-1} \cdot \boldsymbol{F}_{p}$

and a projection of the sensitivity onto the space perpendicular to the ILDM yields (cf. Section 2)

$\hat{\boldsymbol{S}}^{\mathrm{PSR}}=-\boldsymbol{G}_{\psi}^{+} \cdot \boldsymbol{G}_{\psi} \cdot\left(\boldsymbol{F}_{\psi}-\omega \cdot \boldsymbol{I}\right)^{-1} \cdot \boldsymbol{F}_{p}$. 
Using the decomposition of $\boldsymbol{F}_{\psi}$ according to Eq. (2), we obtain

$$
\left.\left.\hat{\boldsymbol{S}}^{\mathrm{PSR}}=-\boldsymbol{G}_{\psi}^{+} \cdot \boldsymbol{G}_{\psi} \cdot \boldsymbol{Z} \cdot\left\{\left(\begin{array}{cc}
\boldsymbol{N}_{\mathrm{s}} & \mathbf{0} \\
\mathbf{0} & \boldsymbol{N}_{\mathbf{f}}
\end{array}\right)-\omega \boldsymbol{I}\right)\right)\right\}^{-1} \cdot \boldsymbol{Z}^{-1} \cdot \boldsymbol{F}_{p} \cdot
$$

If as an approximation we assume only slight variations of $\boldsymbol{Z}$ with varying $\boldsymbol{\psi}, \boldsymbol{G}_{p}$ and $\boldsymbol{G}_{\boldsymbol{\psi}}$ can be written as:

$\boldsymbol{G}_{p} \approx \boldsymbol{Z} \cdot \boldsymbol{F}_{p}$,

$\boldsymbol{G}_{\psi} \approx \boldsymbol{Z} \cdot \boldsymbol{F}_{\psi}$

Insertion of these expressions into the equation for $\hat{\boldsymbol{S}}^{\mathrm{PSR}}$ yields after some calculation steps

$\hat{\boldsymbol{S}}^{\mathrm{PSR}} \approx-\boldsymbol{G}_{\psi}^{+} \cdot \boldsymbol{N}_{\mathbf{f}} \cdot\left(\boldsymbol{N}_{\mathrm{f}}-\omega \cdot \boldsymbol{I}\right)^{-1} \cdot \boldsymbol{G}_{p}$.

Noting that the sensitivity equation for the ILDM is given by the equation

$\boldsymbol{S}=-\boldsymbol{G}_{\psi}^{+} \cdot \boldsymbol{G}_{p}$,

it can be seen that the sensitivity of the ILDM and that of the PSR (after projection) become equivalent for

$|\omega| \ll\left|\lambda\left(\boldsymbol{N}_{\mathbf{f}}\right)\right|$,

i.e., for the mixing time scale being larger than the slowest decoupled time scale. Thus, it can be stated that both the sensitivities of an ILDM and those of a PSR should in most cases have similar underlying features.

To get an idea of the overall sensitivity of the ILDM with respect to all 67 elementary reactions of the syngas-air-system, sensitivities have been calculated for the 2d-ILDM of the system. In Fig. 3 , global relative sensitivities (maximum values over the whole domain of the ILDM) for the radical $\mathrm{OH}$ are shown for the most sensitive reactions. It can be seen that the qualitative behaviour, e.g., the fact that only few reactions are sensitive, is similar to the results of other sensitivity analyses [1,15],

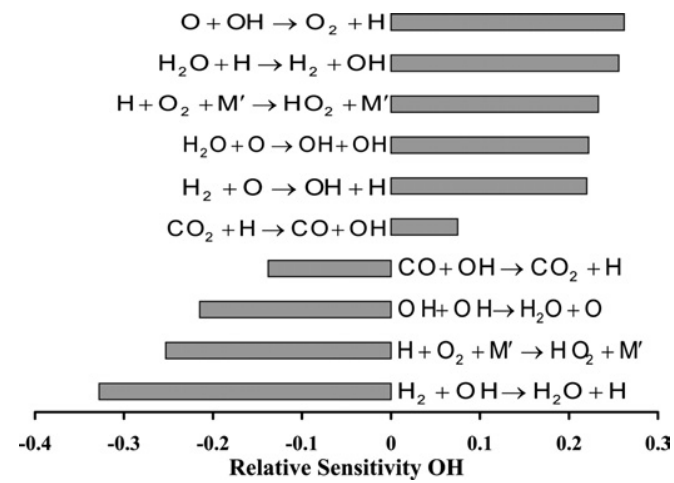

Fig. 3. Sensitivity analysis for the 2d-ILDM of the CO/ $\mathrm{H}_{2} / \mathrm{O}_{2} / \mathrm{N}_{2}$-system. Only the most sensitive reactions with respect to $\mathrm{OH}$ are shown. Sensitivities are given for the $\mathrm{OH}$ concentrations. Species concentrations and sensitivities are given in terms of $\left[w_{\mathrm{i}} / M_{\mathrm{i}}\right]=\mathrm{mol} / \mathrm{kg}$. although, of course, the quantitative behaviour differs. This can be attributed to two effects. First, the sensitivity for the ILDM considers all domains where the ILDM exists, whereas sensitivity analyses, e.g., for ignition processes or flame velocities are related to specific areas of the state space only (e.g., low temperature regime in the case of ignition processes). Second, the sensitivity analysis for the ILDM determines only the sensitivities perpendicular to the ILDM. The sensitivities related to the dynamics of the movement along the ILDM (cf. the relatively low sensitivity for $\mathrm{CO}+\mathrm{OH} \rightarrow$

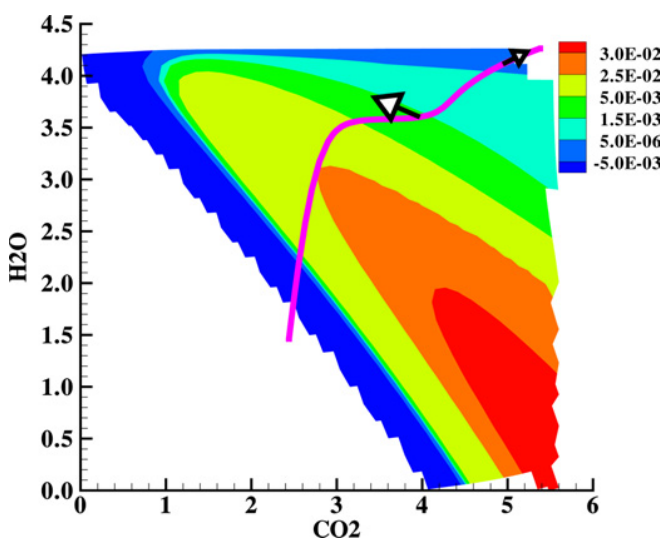

Fig. 4. 2d-ILDM of the $\mathrm{CO} / \mathrm{H}_{2} / \mathrm{O}_{2} / \mathrm{N}_{2}$-system projected into the $\mathrm{CO}_{2}-\mathrm{H}_{2} \mathrm{O}$-plane. Sensitivities of the ILDM for $\mathrm{OH}$ are shown for the reaction $\mathrm{H}+\mathrm{O}_{2}+\mathrm{M} \rightarrow \mathrm{HO}_{2}+\mathrm{M}$. Purple trajectory shows the reaction progress, black arrows show the sensitivity of the trajectory (due to better legibility arrows are scaled with a common factor). Species concentrations and sensitivities are given in terms of $\left[w_{\mathrm{i}} / M_{\mathrm{i}}\right]=\mathrm{mol} / \mathrm{kg}$.

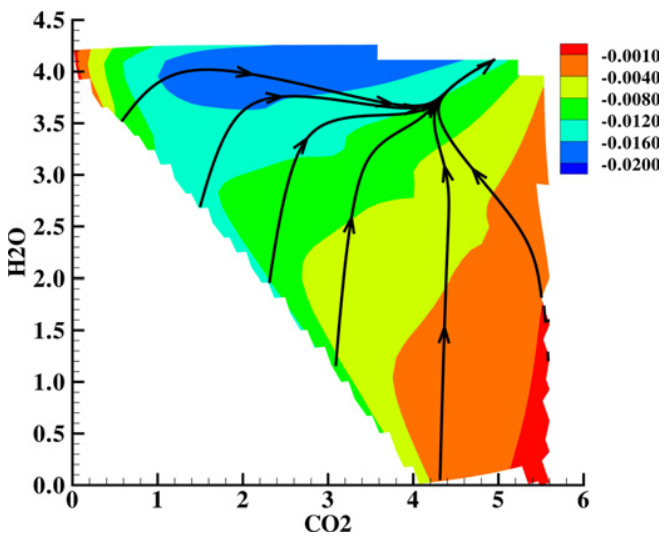

Fig. 5. 2d-ILDM of the $\mathrm{CO} / \mathrm{H}_{2} / \mathrm{O}_{2} / \mathrm{N}_{2}$-system projected into the $\mathrm{CO}_{2}-\mathrm{H}_{2} \mathrm{O}$-plane. Sensitivities of the ILDM for $\mathrm{OH}$ are shown for the reaction $\mathrm{CO}+\mathrm{OH} \rightarrow \mathrm{CO}_{2}+\mathrm{H}$. Trajectories show the reaction progress, the moving towards a 1-dimensional ILDM and the final approach of the chemical equilibrium. Species concentrations and sensitivities are given in terms of $\left[w_{\mathrm{i}} / M_{\mathrm{i}}\right]=\mathrm{mol} / \mathrm{kg}$. 
$\mathrm{CO}_{2}+\mathrm{H}$, which is a rate limiting reaction on the way towards equilibrium but does not affect too much the steady state value of $\mathrm{OH}$ ) are not accounted for (cf. Section 2). Note, however, that this sensitivity of the movement along the ILDM can be obtained by a simple projection of the sensitivity vector $\boldsymbol{F}_{p}$ of $\boldsymbol{F}$ onto the ILDM.

For two reactions with large sensitivities, $\mathrm{H}+\mathrm{O}_{2}+\mathrm{M} \rightarrow \mathrm{HO}_{2}+\mathrm{M}$ and $\mathrm{CO}+\mathrm{OH} \rightarrow$ $\mathrm{CO}_{2}+\mathrm{H}$, the dependence of the sensitivity on the respective domain on the ILDM is now discussed. To simplify the graphical representation, projections into the 2-dimensional- $\mathrm{CO}_{2}-\mathrm{H}_{2} \mathrm{O}$-subspace were chosen. The sensitivities refer to $\mathrm{OH}$ and are given as values of the specific mole numbers $\Phi=w_{\mathrm{OH}} / M_{\mathrm{OH}}$ with $w$ as the mass fraction and $M$ as molar mass. Figure 4 shows the sensitivities for the reaction $\mathrm{H}+\mathrm{O}_{2}+\mathrm{M} \rightarrow \mathrm{HO}_{2}+\mathrm{M}$. This reaction is one of those governing the reaction rate in the initial phase of the reaction process far from equilibrium [1] and, as it can be seen in Fig. 3, it is also governing the behaviour of the ILDM. The sensitivities for the reaction are high for values far away from equilibrium, i.e., for small values of $\mathrm{CO}_{2}$ and $\mathrm{H}_{2} \mathrm{O}$. The more the reaction progress approaches equilibrium, the smaller the sensitivities become, what is evident, because this reaction is of minor importance at high temperatures [1]. Figure 4 in addition, shows a trajectory together with two of its sensitivity vectors to get a comparison of the sensitivity of the ILDM and the sensitivities of a trajectory, corresponding, e.g., to a homogeneous ignition process (note that the vectors have been scaled with a common factor to ensure good legibility). It can be seen that the qualitative behaviour of the trajectory sensitivity is the same as that for the ILDM: It is large in areas where the ILDM has large sensitivities, too, and in the domain near equilibrium, where the sensitivity of the ILDM vanishes, the trajectory sensitivity has only a component parallel to the ILDM. Figure 5 illustrates the sensitivities for the reaction $\mathrm{CO}+\mathrm{OH} \rightarrow \mathrm{CO}_{2}+\mathrm{H}$. This reaction governs the heat release rate [1] and, according to the reaction discussed before, it is also an important reaction for the ILDM. The sensitivities attain their smallest values when close to equilibrium while they have their maximums in the area of high $\mathrm{H}_{2} \mathrm{O}$ and middle to low $\mathrm{CO}_{2}$-values. The reason for the moderate sensitivity of this reaction close to equilibrium has already been discussed above.

\section{Conclusions}

A method for the calculation of sensitivities of ILDMs with respect to kinetic data was introduced and discussed in this paper. After deriving the governing equations, sample results for the stoichiometric $\mathrm{CO} / \mathrm{H}_{2} / \mathrm{O}_{2} / \mathrm{N}_{2}$-system were dis- cussed. A comparison between the points of an ILDM calculated with a perturbed mechanism and the endpoints of sensitivity vectors calculated on the base of an ILDM of the unperturbed mechanism showed the validity of the algorithm, and a 2-dimensional ILDM with its sensitivity vectors gave an overview over the different lengths and directions of the sensitivity vectors. The global relative sensitivities of the 2-dimensional ILDM with respect to $\mathrm{OH}$ were presented, illustrating that the behaviour of the ILDM is qualitative comparable to the behaviour of detailed mechanisms. A closer look at two of the most sensitive reactions offered values of the sensitivities and showed their distribution over the ILDM. Summarizing, the calculated sensitivities do not only tell which reactions are very sensitive, but, in addition, they show the domains of the ILDM in which these reactions have a high sensitivity. As a consequence, the model introduced in this paper allows one to estimate the influence of a change in the detailed mechanism onto the ILDMs. It shall be noted that the method derived in this paper can be applied to ILDMs of other chemical systems (e.g., higher hydrocarbon combustion) and to ILDMs of arbitrary dimensions.

\section{References}

[1] J. Warnatz, U. Maas, R.W. Dibble, Combustion, Physical and Chemical Fundamentals, Modeling and Simulation, Experiments, Pollutant Formation. Springer-Verlag, Berlin, Heidelberg, 2001.

[2] C. Chevalier, J. Pitz, J. Warnatz, C.K. Westbrook, H. Melenk, Proc. Combust. Inst. 24 (1992) 93-101.

[3] U. Maas, S.B. Pope, Combust. Flame 88 (1992) 239-264.

[4] U. Maas, S.B. Pope, Proc. Combust. Inst. 24 (1992) 103-112.

[5] R.L.G.M. Eggels, L.P.H. de Goey, Combust. Flame 100 (1995) 559-570.

[6] O. Gicquel, D. Thevenin, M. Hilka, N. Darabiha, Combust. Theor. Model. 3 (1999) 479-502.

[7] C. Correa, H. Niemann, B. Schramm, J. Warnatz, Proc. Combust. Inst. 28 (2000) 1607-1614.

[8] R.T. Skodje, M.J. Davis, J. Phys. Chem. A 105 (45) (2001) 10356-10365.

[9] T. Blasenbrey, U. Maas, Proc. Combust. Inst. 28 (2000) 1623-1630.

[10] J. Nafe, U. Maas, Proc. Combust. Inst. 29 (2002) 1379-1385.

[11] U. Maas, Comp. Vis. Sci. 1 (1998) 69-82.

[12] G.H. Golub, C.F. van Loan, Matrix Computations. The John Hopkins University Press, Baltimore and London, 1996.

[13] K. König, U. Maas (in press).

[14] A.S. Tomlin, T. Turanyi, M.J. Pilling, in: M.J. Pilling (Ed.), Mathematical Tools for the Construction, Investigation and Reduction of Combustion Mechanisms. Elsevier, Amsterdam, 1997.

[15] U. Maas, J. Warnatz, Proc. Combust. Inst. 22 (1988) 1675-1704. 


\section{Comments}

Dirk Roekaerts, Delft University of Technology, Netherlands. Can the sensitivity analysis you described be useful to decide whether it is necessary to increase the dimensionality of the ILDM to reach certain accuracy? In other words, could there be a link between low sensitivity and relatively high accuracy?

Reply. The sensitivity analysis introduced in this paper does not provide information on the necessary dimension of the ILDM. This dimension is governed by the magnitude of the physical perturbations that tend to pull the system off the manifolds. On the other hand the methodology derived in the paper can be generalized to handle sensitivities with respect to perturbations of the chemical kinetics and such an analysis would indeed yield information on the necessary dimension of the ILDM.

Tamas Turanyi, Eötvös University (ELTE), Hungary. 1. In a recent article [1] we show that local similarity of the sensitivity functions of a model based on a detailed reaction mechanism may emerge if the tangential sensitivity of the corresponding ILDM is much larger than its perpendicular sensitivity. Your present calculations allow a direct comparison of these two terms. Have you made such a comparison?
2. The lecture concentrated on the sensitivity of the displacement of the ILDM to the parameters of the kinetic model. For the application of an ILDM as a reduced chemistry model, the sensitivity of the rate of the parameterising variables to the ILDM is also of interest and these two types of sensitivity information should be handled together.

\section{Reference}

[1] I.Gy. Ziely, F. Zádor, T. Turanyi, J. Phys. Chem. A (2003).

Reply. 1. No, we have not yet made such a comparison but we are planning to investigate this behavior in future work

2. Indeed, the present work deals only with the sensitivity of the ILDM itself. The overall sensitivity of the solution of the reduced model with respect to the rates tangential to the manifold depends on the initial conditions of the reaction trajectories and is thus no longer an intrinsic property of the ILDM. It can, however, be calculated with standard techniques for solving the sensitivity equations of the reduced scheme provided that the sensitivities of the tangential rates with respect to the location of the manifold are calculated together with the sensitivities of the manifold. This will be the subject of future work. 